\title{
PATHOLOGICAL MOTION DETECTION FOR ROBUST MISSING DATA TREATMENT IN DEGRADED ARCHIVED MEDIA
}

\author{
David Corrigan, Naomi Harte, Anil Kokaram* \\ Department of Electronic and Electrical Engineering, \\ University of Dublin, Trinity College, \\ Dublin, Ireland.
}

\begin{abstract}
This paper outlines an algorithm to improve the robustness of Missing Data Treatment to Pathological Motion (PM). PM can cause misdiagnosis of clean image data as missing data. The proposed algorithm uses a probabilistic framework to jointly detect PM and missing data by exploiting more temporal information than is typically used for Missing Data Detection and by exploiting the local smoothness assumption of motion fields. The results of the framework are compared to an equivalent missing data detector without PM detection and the framework is shown to prevent the misdiagnosis of missing data due to PM.
\end{abstract}

Index Terms - Image Restoration, Image Motion Analysis

\section{INTRODUCTION}

Motion estimation is key to a wide variety of digital video processing tasks. Unfortunately, there are a number of situations in which motion cannot be estimated regardless of the complexity of the estimator. Two of the most common causes of motion estimation failure are Missing Data (regions of damaged or destroyed image data typically caused by dirt and sparkle [1]) and Pathological Motion (PM) (object motion that is impossible to estimate). Forms of PM include occlusion and uncovering of objects, intermittent motion, motion blur and self-occluding motion [2]. Long-term PM, where PM persists over a number of frames, causes false alarms in missing data detection (see figure 1). Missing Data is modeled as a region of image data which is present in a single frame only. Hence it is typically detected by observing temporal discontinuities in the image intensity function over a 3 frame window centred on the current frame [1,3]. Longterm PM causes false alarms since it will also cause temporal discontinuities over a 3 frame window and be mistaken for missing data.

Previous approaches for dealing with Pathological Motion have largely fallen into two categories. The first does

*Thanks to the Irish Research Council for Science, Engineering and Technology for funding this research.

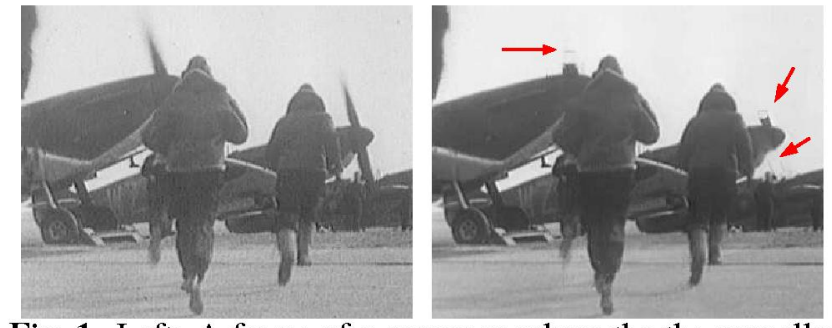

Fig. 1. Left: A frame of a sequence where the the propeller blades exhibit PM; Right: The image after missing data treatment [3]. The arrows indicate the removal of the blades due to PM being misdiagnosed as missing data.

not involve detecting PM directly but rather detecting regions which are likely to contain PM and then to make Missing Data Treatment more conservative in these areas [4]. An alternative strategy is to firstly detect all regions of motion estimation failure and then to classify between missing data and PM [2, 5]. Bornard proposed in [5] that long-term PM could be detected by searching for temporal discontinuities over a number of frames. Given the motion information for a sequence, a probabilistic framework is used to estimate the 4 binary temporal discontinuity fields over a 5 frame window. This is followed by a deterministic interpretation step to perform the classification. Missing data is classified if temporal discontinuities only exist at a site in the 2 central discontinuity fields. Sites at which discontinuities also exist in the two outer fields are classified as PM.

In this paper a new probabilistic framework for long-term PM detection is proposed. The framework extends on Bornard's observation that PM can be detected using more temporal information by incorporating the observation that PM causes the local smoothness assumption of a motion field to be violated. A more complete frame model is used considering every possible configuration of the 4 temporal discontinuity fields (16 in total). Each configuration is modeled as either PM or missing data allowing both PM and missing data to be detected. Unlike Bornard's approach, there is no interpretation step as the framework directly detects PM from the 5 frame window and motion information. This allows prior information to be incorporated into the final result rather than 
the temporal discontinuity fields.

The next section describes in more detail the frame model and the operation of the framework. In the following section, experimental results for a number of sequences are presented. Also a simple missing data treatment experiment is described that demonstrates that the proposed framework provides missing data detection robust to PM.

\section{PATHOLOGICAL MOTION DETECTION}

The proposed PM detection algorithm uses a probabilistic framework to detect PM in a frame given the frame and the two previous and subsequent frames as well as the motion information for the 5 frames. Any motion estimator that outputs results expressible as a vector field (e.g. [1]) can be used to generate the motion information. This section firstly describes the context for the framework and then the probabilistic framework itself. The final subsection provides some information on how the detection is performed in practice.

\subsection{Temporal Discontinuity Estimation}

Temporal discontinuities are estimated using the motion compensated Displaced Frame Differential (DFD). For a DFD $\Delta_{k}$, there is an associated binary Temporal Discontinuity Field $t_{k}$, where pixels with high DFD values are indicative of temporal discontinuities. In total 4 DFDs are calculated between consecutive frames in the 5 frame window and are given as follows

$$
\begin{aligned}
& \Delta_{n-2}(\mathbf{x})=I_{n-1}\left(\mathbf{x}+\mathbf{d}_{n}^{b}\right)-I_{n-2}\left(\mathbf{x}+\mathbf{d}_{n}^{b}+\mathbf{d}_{n-1}^{b}\right) \\
& \Delta_{n-1}(\mathbf{x})=I_{n}(\mathbf{x})-I_{n-1}\left(\mathbf{x}+\mathbf{d}_{n}^{b}\right) \\
& \Delta_{n+1}(\mathbf{x})=I_{n}(\mathbf{x})-I_{n+1}\left(\mathbf{x}+\mathbf{d}_{n}^{f}\right) \\
& \Delta_{n+2}(\mathbf{x})=I_{n+1}\left(\mathbf{x}+\mathbf{d}_{n}^{f}\right)-I_{n+2}\left(\mathbf{x}+\mathbf{d}_{n}^{f}+\mathbf{d}_{n+1}^{f}\right)
\end{aligned}
$$

where $\Delta(\mathbf{x})$ is the DFD value at pixel site $\mathbf{x}$. The quantities $\mathbf{d}_{n}^{f}$ and $\mathbf{d}_{n}^{b}$ are the forward and backward motion vector fields for frame $n$ (i.e. the motion from frame $n$ to $n+1$ and $n$ to $n-1)$ respectively and $I_{n-2}, I_{n-1}, I_{n}, I_{n+1}$ and $I_{n+2}$ are the intensity functions of the 5 frames of the temporal window. It should be noted that all frames are motion compensated with respect to the central frame $I_{n}$.

\subsection{The State Model}

The goal of the algorithm is to segment the central frame into regions of $\mathrm{PM}$, missing data and uncorrupted sites. To achieve this, a label field is defined as follows

$$
l(\mathbf{x})= \begin{cases}0 & \text { No missing data or Pathological Motion } \\ 1 & \text { Missing Data } \\ 2 & \text { Pathological Motion. }\end{cases}
$$

\begin{tabular}{|c|c|c||c|c|c|}
\hline State & $\mathbf{s}(\mathbf{x})$ & $l(\mathbf{x})$ & State & $\mathbf{s}(\mathbf{x})$ & $l(\mathbf{x})$ \\
\hline 0 & $0,0,0,0$ & 0 & 8 & $1,0,0,0$ & 0 \\
1 & $0,0,0,1$ & 0 & 9 & $1,0,0,1$ & 2 \\
2 & $0,0,1,0$ & 0 & 10 & $1,0,1,0$ & 2 \\
3 & $0,0,1,1$ & $2^{*}$ & 11 & $1,0,1,1$ & 2 \\
4 & $0,1,0,0$ & 0 & 12 & $1,1,0,0$ & $2^{*}$ \\
5 & $0,1,0,1$ & 2 & 13 & $1,1,0,1$ & 2 \\
6 & $0,1,1,0$ & 1 & 14 & $1,1,1,0$ & 2 \\
7 & $0,1,1,1$ & 2 & 15 & $1,1,1,1$ & 2 \\
\hline
\end{tabular}

Table 1. The state model for the probabilistic framework. * States 3 and 12 can correspond to missing data in the neighbouring frames. It is considered here as PM as it is not caused by missing data in the current frame.

In order to perform the segmentation, it is necessary to estimate the temporal discontinuity values of each discontinuity field at a site. There are 16 possible temporal discontinuity configurations since 4 discontinuity field values are associated with each site. Each configuration is directly mapped to a value of $l(\mathbf{x})$. If missing data is present, temporal discontinuities exist only in the central discontinuity fields and therefore the configuration for the site, given by $\mathbf{s}(\mathbf{x})=\left[t_{n-2}(\mathbf{x})\right.$, $\left.t_{n-1}(\mathbf{x}), t_{n+1}(\mathbf{x}), t_{n+2}(\mathbf{x})\right]$, is $0,1,1,0$. Long-term PM is given by any configuration or state where at least two discontinuities exist except for $0,1,1,0$. A full description of the state model and their mappings to $l(\mathbf{x})$ is given in Table 1 .

\subsection{Pathological Motion and Motion Field Smoothness}

PM often violates the local smoothness assumption of a motion field. A useful measure of smoothness in a vector field is the divergence. High divergence values correspond to regions of the field which are not smooth and so may be associated with PM. Therefore high divergence values can occur in a series of motion fields if long-term PM exists. For the purposes of this algorithm, a deterministic thresholding operation is used to detect high divergences in the motion fields used for motion compensation. Firstly the sum of the absolute divergence values for the motion fields is calculated and then a threshold (50 in all cases) is applied. The result is a binary divergence mask $b_{d i v}(\mathbf{x})$, where values of $b_{d i v}(\mathbf{x})=1$ correspond to sites at which PM is suspected.

\subsection{The Probabilistic Framework}

In order to obtain a solution for frame $n$, a solution for $\mathbf{s}(\mathbf{x})$ and $l(\mathbf{x})$ must be found given the temporal discontinuity and divergence information. This is achieved by manipulating the posterior $p\left(l, \mathbf{s} \mid \boldsymbol{\Delta}_{n}, b_{d i v}, L\right)$ using Bayes' Law as follows

$$
p\left(l, \mathbf{s} \mid \boldsymbol{\Delta}_{n}, b_{d i v}, L\right) \propto p\left(\boldsymbol{\Delta}_{n} \mid l, \mathbf{s}\right) p(l \mid L) p\left(l \mid b_{d i v}\right) .
$$

For convenience, the index $\mathrm{x}$ has been omitted and the 4 DFDs have been grouped into a vector valued function $\boldsymbol{\Delta}_{n}$ 
where $\Delta_{\mathbf{n}}(\mathbf{x})=\left[\Delta_{n-2}(\mathbf{x}), \Delta_{n-1}(\mathbf{x}), \Delta_{n+1}(\mathbf{x}), \Delta_{n+2}(\mathbf{x})\right]$. $L$ is set of values $l(\mathbf{y})$ for all $\mathbf{y}$ in a neighbourhood of $\mathbf{x}$.

The data likelihood $p\left(\boldsymbol{\Delta}_{n} \mid l, \mathbf{s}\right)$ constrains each DFD to be low when a temporal discontinuity does not exist according to eq. 1 . The expression for the likelihood is

$$
p\left(\boldsymbol{\Delta}_{n} \mid l, \mathbf{s}\right) \propto \exp -\sum_{k=0}^{3}\left\{\frac{\boldsymbol{\Delta}_{\mathbf{n}}[k]^{2}}{2 \sigma_{e}^{2}}(1-\mathbf{s}[k])+\alpha \mathbf{s}[k]\right\}
$$

where $\mathbf{v}[k]$ is the $k^{\text {th }}$ component of a vector $\mathbf{v}, \sigma_{e}^{2}$ is the variance of $\boldsymbol{\Delta}_{\mathbf{n}}$ at uncorrupted sites and $\alpha$ is a probability penalty for temporal discontinuity associated with the $99 \%$ confidence level of a gaussian distribution $\left(\alpha=2.76^{2} / 2\right)$.

Two probability priors are associated with this framework. The first, $p(l(\mathbf{x}) \mid L)$, is a spatial smoothness constraint derived from the observation that PM and missing data manifests as contiguous regions rather than isolated sites. A Gibbs Energy Prior [6] is used and is given by

$$
p(l \mid L) \propto \exp -\left\{\Lambda_{l} \sum_{\mathbf{y} \in \mathcal{N}(\mathbf{x})} \lambda_{\mathbf{y}}|l(\mathbf{x}) \neq l(\mathbf{y})|\right\}
$$

where $\Lambda_{l}$ is the weight of spatial smoothness (typically $\Lambda_{l}=$ $1), l_{y}$ is a weight inversely proportional to $|\mathbf{x}-\mathbf{y}|$ and $\mathcal{N}(\mathbf{x})$ is the 8-connected neighbourhood of $\mathbf{x}$. A smoothness prior is not associated with $\mathbf{s}(\mathbf{x})$ for reasons of computational complexity.

The second prior is a data driven prior which enforces the constraint that PM is associated with large motion field divergences. It constrains $l(\mathbf{x})$ (and $\mathbf{s}(\mathbf{x})$ ) to be a PM state when $b_{\text {div }}=1$. The expression for the prior is

$$
p\left(l \mid b_{d i v}\right) \propto \exp -\Lambda_{b} \times \begin{cases}b_{d i v} & l \neq 2 \\ \left(1-b_{d i v}\right) & l=2\end{cases}
$$

where $\Lambda_{b}$ is the weight associated with the prior (typically $\Lambda_{b}=1$ ).

\subsection{A practical solution}

There are 16 possible solutions associated with $\mathrm{s}(\mathrm{x})$ at each pixel. The solution for $l(\mathbf{x})$ is then obtained directly from table 1. A result is obtained by evaluating the posterior for each configuration of $\mathrm{s}$ and then choosing the MAP estimate for each site. This is achieved using the ICM algorithm [7]. This process is then iterated and iterations continue until the result converges up to a maximum of 20 iterations. A checkerboard scan is used to reduce the propagation of errors. The multiresolution scheme described by Heitz et al. [8] is employed whereby the result at a coarser resolution, obtained by subsampling the DFDs and divergence mask, is used to provide an initial estimate for $\mathrm{s}$ and $l$ at a finer resolution.

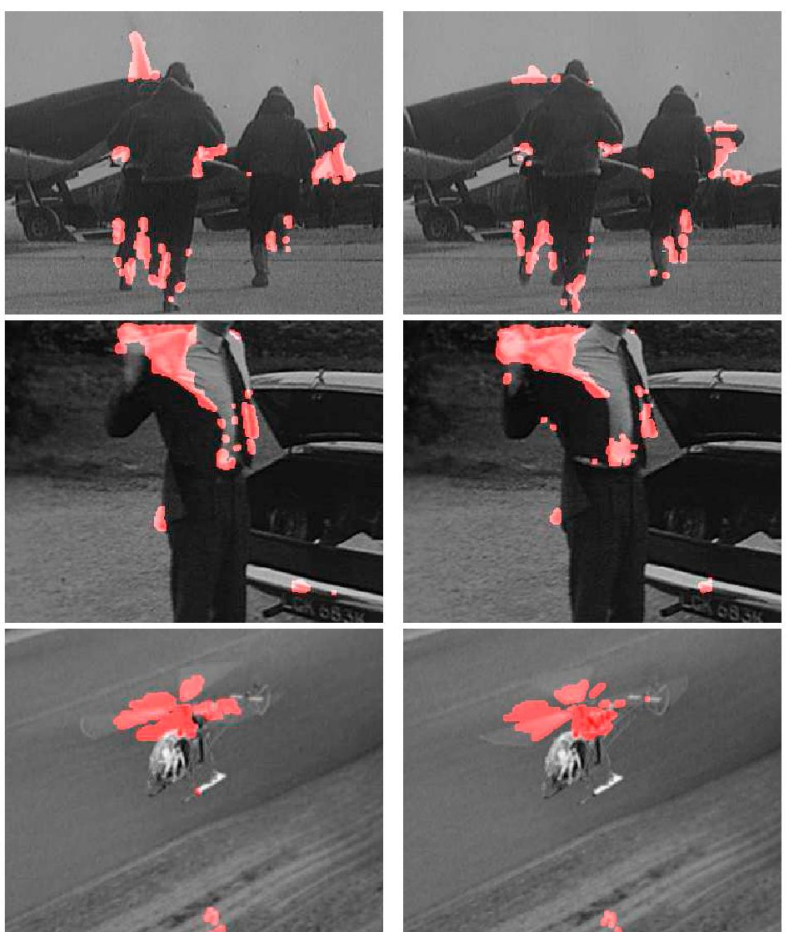

Fig. 2. Each row show consecutive frames from the sequences discussed in Section 3. The detected PM regions are highlighted. In the top sequence the intermittent PM of the propellers and the self-occluding motion of the airmen are detected. The self-occluding motion of the jacket in the $2^{\text {nd }}$ sequence and the blurred motion of the rotors in the $3^{\text {rd }}$ are detected.

\section{EXPERIMENTAL RESULTS}

Pathological Motion detection results are shown here for 3 test sequences, each exhibiting a different form of PM. The first sequence (fig. 2, top row) is an example of intermittent PM caused by the repetitive occlusion and uncovering of the propeller blades, the second (fig. 2, middle row) is an example of self-occluding motion (the motion of the jacket) and the final example (fig. 2, bottom row) contains motion blur due to the quick motion of the rotor blades. The results show that the framework detects the various forms of PM shown in the sequences.

An experiment was undertaken to determine the effect of the PM detection framework on the performance of missing data treatment. As the PM detection framework also models missing data, it can be used to detect missing data. The framework was compared with a missing data detector with no PM detection (based on the tear delineation algorithm in [9]). The results from both detectors were used to initialise a simple missing data recovery technique by interpolating detected missing data from the previous and next frames. Results from the experiment are shown in figure 3 . The destruction of image data, seen in the results of missing data treatment without PM detection, has largely been avoided when 

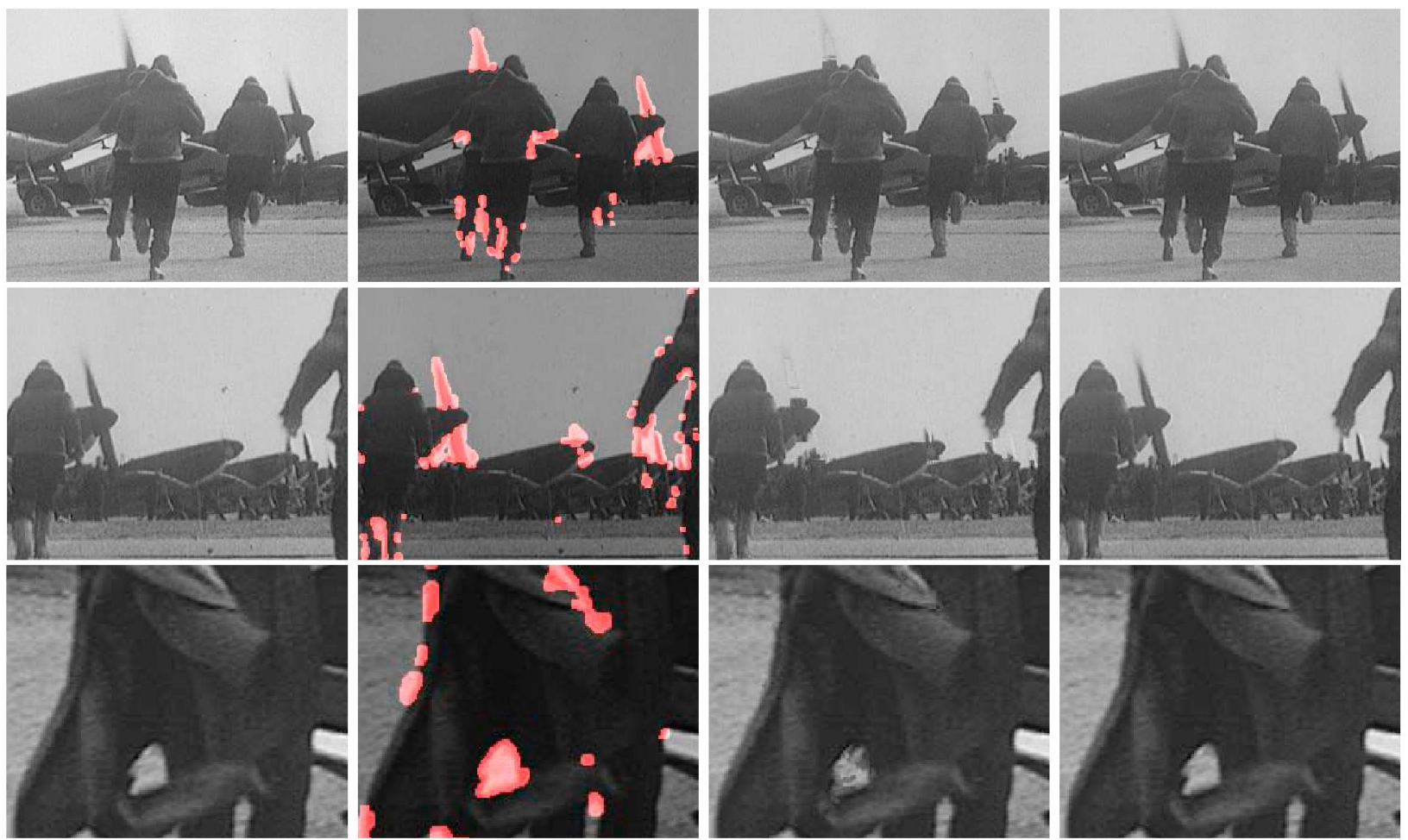

Fig. 3. This figure shows the results of the missing data treatment experiment for 3 frames. Far Left Column: A zoom of the 3 original frames; Middle Left: Detected PM highlighted; Middle Right; The result of missing data removal without PM detection; Far Right: The result of missing data treatment using the Long-term PM Detector. The destruction of image data has been avoided using the proposed framework. Missing data apparent in the frame on the $2^{\text {nd }}$ row is removed by both frameworks.

the PM detection framework is used to detect missing data.

\section{CONCLUDING REMARKS}

In this paper a probabilistic framework has been presented that detects long-term Pathological Motion from 5 frames of image data and the corresponding motion information. Every possible temporal discontinuity configuration over the 5 frames is modeled. This, along with the use of the divergence, prevents the misdiagnosis of PM as missing data and has been demonstrated in fig. 3. Work is ongoing to compare the framework with other PM detection methods (most notably [5]) and to integrate the framework into an existing missing data treatment technique making it robust to PM.

\section{REFERENCES}

[1] Anil Kokaram, Motion Picture Restoration: Digital Algorithms for Artefact Suppression in Degraded Motion Picture Film and Video, Springer Verlag, 1998.

[2] Andrei Rares, Archived Film Analysis and Restoration, Ph.D. thesis, Delft University of Technology, 2004.

[3] Anil Kokaram and Simon Godsill, "Mcmc for joint noise reduction and missing data treatment in degraded video,"
IEEE trans. on Signal Processing, vol. 50, pp. 189-205, February 2002.

[4] B. Kent, A. Kokaram, B. Collis, and S. Robinson, "Two layer segmentation for handling pathological motion in degraded post production media," in IEEE Int. Con. on Image Processing (ICIP), Singapore, 2004.

[5] R. Bornard, Probabilistic Approaches for the Digital Restoration of Television Archives, Ph.D. thesis, Ecole Centrale Paris, 2002.

[6] J. Konrad and E. Dubois, "Bayesian estimation of motion vector fields," IEEE trans.on Pattern Analysis and Machine Intelligence, vol. 14, September 1992.

[7] J.E. Besag, "On the statistical analysis of dirty pictures," Journal of the Royal Statistical Society B, vol. 48, pp. 259-302, June 1986.

[8] F. Heitz, P. Perez, and P. Bouthemy, "Multiscale minimization of global energy functions in some visual recovery problems," CVGIP: Image Understanding, vol. 59, pp. 125-134, January 1994.

[9] D. Corrigan and A. Kokaram, "Automated tear treatment in degraded archived media," in IEEE ICIP, Singapore, 2004. 